\title{
Technical and Scientific Breakthroughs Highlighted
}

Some 3100 posters and talks were presented at the joint EPS condensed matter/German Physical Society solid-state meeting, Regensburg, Germany, on 29 March - 2 April 1993. The Editor summarises notable contributions.
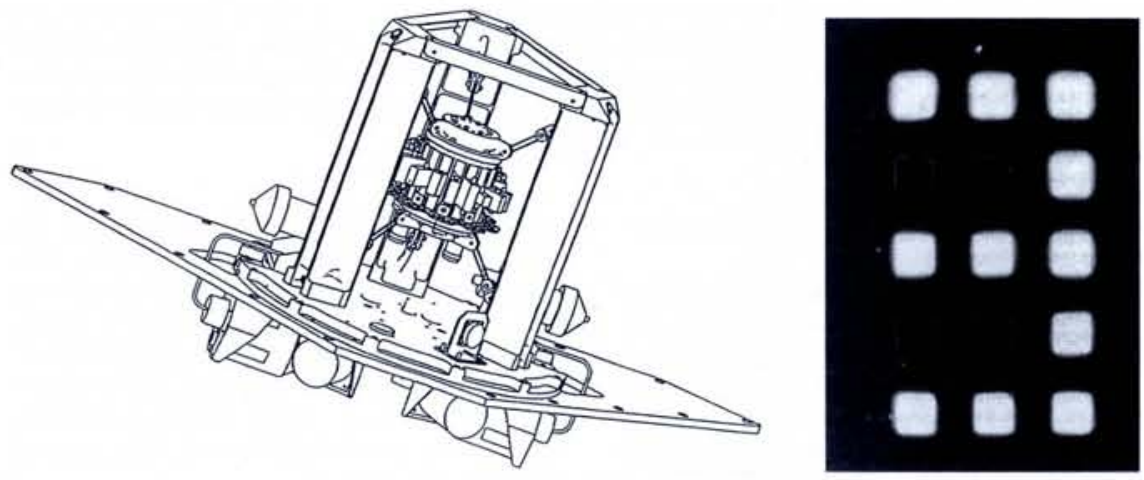

Technical highlights of the Regensburg meeting: on the left, an illustration of the US Naval Research Laboratory's HTSSE-I satellite that will be launched this year to test high-performance microwave and millimetre wave communications technology. HTSSE-I will contain 11 types of high- $T_{c}$ superconducting devices made by 23 suppliers. A second satellite (HTSSE-II) scheduled for launching in 1994-95 will test components and sub-systems; a totally high- $T_{c}$ spacecraft may follow around the year 2000. Europe has no equivalent programme and is not involved at this stage.

On the right, Cambridge University's polymer light-emitting diode display.

When some 4000 condensed matter physicists meet for a week at what was once called the crossroads of Europe one should anticipate an exciting event. Expectations may have been surpassed as the 13th $\mathrm{Ge}$ neral Conference of the Condensed Matter Division (CMD), held in conjunction with the annual meeting of the Solid-State Physics Arbeitskreis of the German Physical Society (GPS), offered a panoramic view of solidstate physics across a huge range of "scales". There was everything from provocative overviews of novel approaches, to reports of the status of basic technologies in revolutionary new fields, to lively symposia on the latest scientific developments in several topics of current interest. As the meeting followed almost immediately after the American Physical Society's March Meeting in Seattle there is a temptation to use the occasion to make trans-Atlantic comparisons. But that is another story.

H. Rietschel (KfK, Karlsruhe) reviewed the many likely applications of high-temperature superconductors.

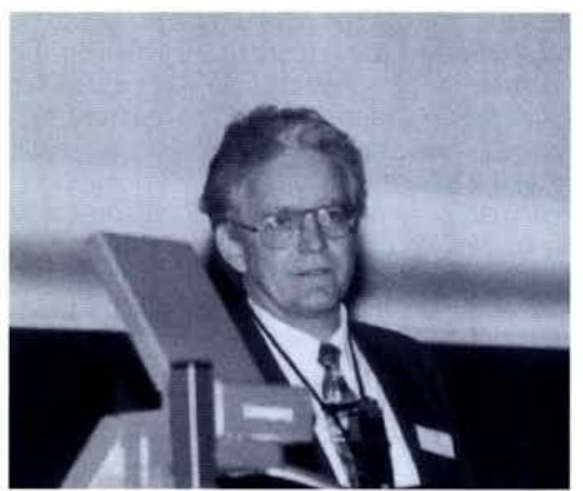

The 171 invited talks, the 1480 contributed talks and the 1441 poster presentations were divided between the 10 GPS divisions which are virtually mirror images of the EPS CMD sections. Excellent organization under Professor M. Schwoerer (University of Bayreuth) as the Conference Chairman, Professor R. Klien (University of Constance) as the Programme Chairman, and Professor H. Hoffman (University of Regensburg) as the Local Chairman, and the close-knit configuration of the conference site in the University of Regensburg's multi-purpose main buildings, ensured that most presentations on important topics spanning several divisions could be attended.

\section{Strategic Importance of Research}

Of the four plenary talks, that by R.R. Ernst (ETH Zurich) - the 1992 Nobel laureate in chemistry - on NMR went beyond the boundaries of physics to give a remarkable review of how a technique, with its origins at the heart of physics, has been developed these last twenty years as a precision probe for atomic and molecular structures essentially throughout natural science. Two of the other plenaries fell more within today's boundaries of condensed matter physics, with T. Springer (KfA Jülich) describing contributions from neutron scattering and S.A. Orszag (Princeton University) reviewing the state-of-the art of turbulent flow simulations.

The remaining plenary talks addressed major industrial developments based directly on discoveries and techniques generated by basic research. Although several major companies and many research groups have apparently lost their enthusiasm for high- $T_{c}$ superconductors and have withdrawn from the field, H. Rietschel (KfK, Karlsruhe) re- dressed the balance by demonstrating an impressive array of actual applications to support his conviction that high- $\mathrm{T}_{c}$ 's are "on their way to becoming a future key technology". Particularly impressive was a US project to launch a series of satellites to test components and subsequently systems. J.-M. Dubois (Ecole des Mines, Nancy) described how quasicrystals, identified a few years ago, are not simply "crystallographic monsters" halfway between metallic glasses and conventional intermetallic compounds. They in fact offer a broad range of unusual properties with promising applications in aerospace, the automotive sector, and cookware. The last plenary talk (G. Abstreiter, University of Munich) showed how Si/Ge heterostructures and multilayers to "engineer" band gaps to integrate, for example, optical devices with Si-based technology, were eventually meeting expectations after some 10 years of intensive work. The breakthrough came within the last few years from the ability to fabricate good quality materials.

\section{Polymer Electronics:}

\section{From Displays to Molecules}

An invited talk in the macromolecular physics division by R.H. Friend (Cambridge University) on the development of electroluminescent polymeric devices also highlighted the strategic importance of basic research. A layer of poly(p-phenylenevinylene) is sandwiched between a metallic electron-injecting electrode and an indium/tin oxide hole-injection electrode. Light emission arises from the radiative decay of an exciton formed by the combination of the injected, oppositely charged polarons. Efficiencies of a few percent have already been obtained, equivalent to a brightness in excess of those for conventional cathode-ray tubes so applications for flat-screen displays are obvious. The technological interest means that physics aspects of electroluminescent polymers have acquired a major new focus. While these aspects were not a major topic at the conference, work by M. Schwoerer's group at Bayreuth illustrated the progress that has been made in fabricating good quality, large-area films, and in investigating the all-important stability aspects.

The idea that an organic molecular layer can act as a p-n junction was proposed in 1974 but it is only recently that the effect seems to have been observed (J.R. Sambles, Exeter University). While the origins of some experimental observations remain slightly puzzling, there is little doubt that the

J.-M. Dubois (Ecole des Mines, Nancy) described potential applications, both industrial and consumer, of quasicrystals.

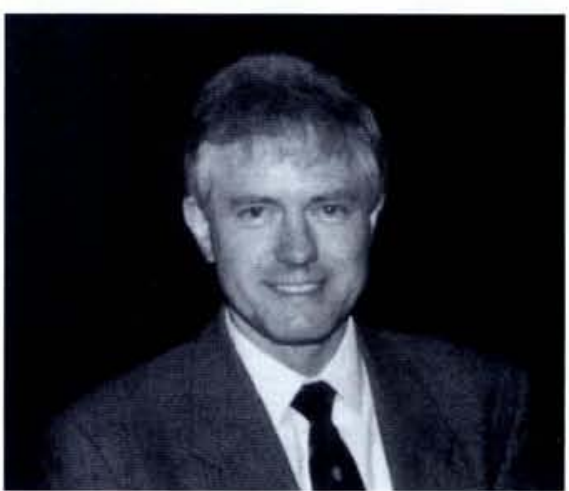


field of molecular rectification, so important for molecular electronics, has received a huge boost.

Moving on down in scale, the use of the scanning tunnelling techniques as a tool to electronically probe complex molecules has been frustratingly slow up to now. J.P. Rabe (Max-Planck, Mainz) described rational approaches for carrying out physics, albeit cautiously, at the molecular level. He focussed on monolayers self-assembled at surfaces and interfaces to show that images can be interpreted in terms of molecular structures.

\section{Handling Intermittency in Turbulence}

D. Lohse (Philipps University, Marburg) in an invited talk in the liquids session on turbulence discussed how intermittency of energy dissipation in three-dimensional turbulence leads to a failure of the Kolmogorov theory based on dimensional arguments (the theory assumes that velocity differences on a scale $r$ only depend on the energy dissipation rate averaged over a sphere of radius $r$ ). The Marburg group has tackled the intermittency problem by finding approximate solutions to the Navier-Stokes equation (exact solutions would require $10^{19}$ grid points) using Fourier transforms with a geometrically scaling subset of wave vectors (some 3098 wave vectors are used, the largest being about the size of the conference centre). The approach predicts the main features of fully developed turbulent flow. For example, the probability distribution function of the velocity differences on a scale $r$ are shown to loose similarity as the scale is reduced. This effect, called small-scale intermittency, arises owing to competition between turbulent transport and viscous damping. The surprising result is that three sub-ranges can be identified: the stirring sub-range with slight intermittency (as observed in smoke plumes), the inertial subrange where there is no intermittency and the viscous sub-range where intermittency is strong since the time scale for viscous damping is much less than the time to dissipate turbulent energy.

Doubts are cast on Kolmogorov's similarity hypothesis because the $r$-scaling of the moments of the locally averaged energy dissipation is non-zero, even for a Gaussian probability of the velocity gradients. An alternative scaling, which may hold in other fields, is proposed where the moments scale with the degree of the non-Gaussian character of the

The complex, multiple-scale structure of turbulent flow revealed by passing a sheet of laser light across a turbulent jet seeded with a dye.

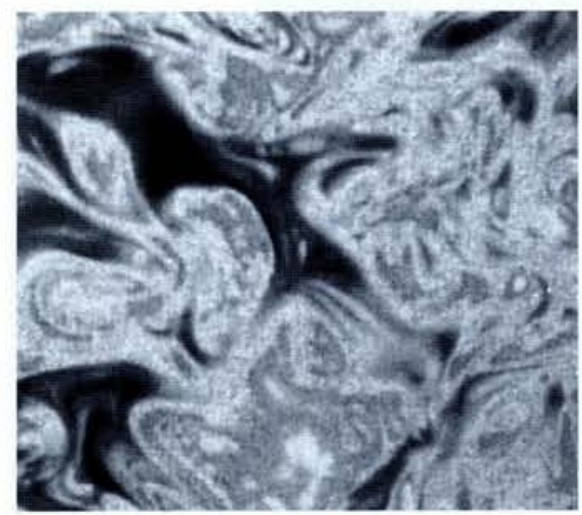

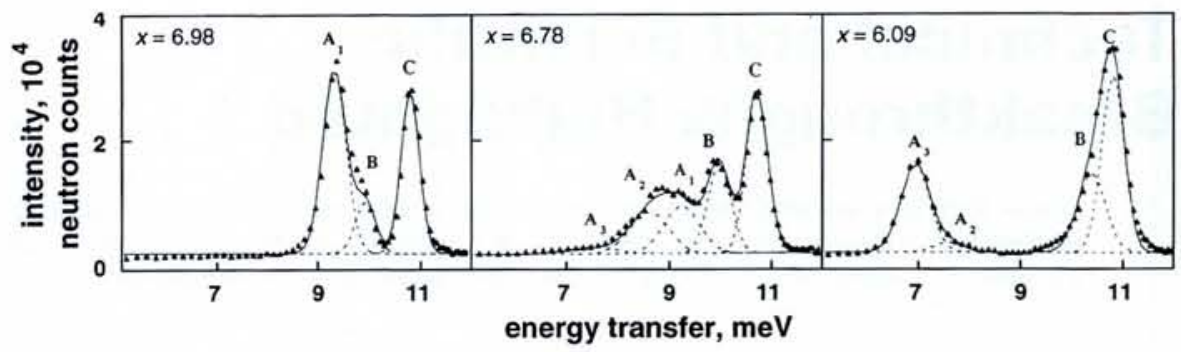

Neutron spectroscopic evidence for the formation of three types of clusters (two metallic, one semiconducting) in the superconducting $\mathrm{CuO}_{2}$ planes of a $\mathrm{LaSrCuO}$ high-T $\mathrm{T}_{c}$ compound. Energy spectra of inelastically scattered neutrons reveal three sub-peaks accompanying the transition from a superconducting to a non-superconducting state on reducing the oxygen stoichiometry $x$ from 7 to 6. [Mesot J., et al., Phys. Rev. Lett. 70 (1993) 865]

probability distribution of the observed velocity difference.

A. Vulpiani (University of Rome) presented an alternative approach to the scaling problem. A shell model for fully developed turbulence is used and the intermittent structure of energy dissipation is allowed for by calculating structure functions to estimate corrections to the Kolmogorov theory. Intermittency is described in terms of the scaling of the multifractality of energy dissipation.

\section{DFT Tackles Basic Problems}

G. Senatore (University of Trieste) described a recently proposed natural extension of the classical density functional theory (DFT) for the freezing of quantum fluids. DFT exploits the fact that a many-body system can be described by a suitable thermodynamic potential, so shifting the emphasis from complicated many-body effects to the much simpler one-body density. It is then natural to investigate crystallization where the density takes on a periodic form. However, implementing DFT requires suitable approximations to thermodynamic potentials: quantum Monte Carlo calculations were used for this. Two-dimensional Wigner electron crystallization has also been treated as it is computationally less demanding, and has practical and scientific significance.

FDT was also used by D.W. Oxtaby (Chicago University) to develop a non-classical theory of nucleation that aims to get around the assumptions of a bulk free-energy difference and a curvature-independent surface term.

An invited talk by M. Scheffler et al. (Fritz Haber Institute, Berlin) in the surfaces, interfaces and thin films section showed how DFT calculations allow the rôles of surface steps on adsorption, vacancy formation, surface diffusion, and crystal growth to be quantified. One surprising result is a revised interpretation of the mechanism for alkali adsorption on aluminium surfaces, where calculations show that the adsorption is activated by surface steps and vacancies. In general, the progress in simulating complex and extended defects is of great benefit to semiconductor technology.

\section{Liquid Helium Continues to Intrigue}

Weakly interacting elementary excitations, essentially rotons and photons, are used to model many properties of liquid ${ }^{4} \mathrm{He}$. However, a theory for finite temperatures is impaired by the lack of a simple and accurate description of the roton excitation. To better understand rotons, A.F.G. Watt (Exeter Uni- versity) described experiments involving the injection of the excitations into liquid ${ }^{4} \mathrm{He}$. High-energy phonons and rotons were found to be extremely well defined (having infinite they interact with the ambient photons and are scattered in four-particle processes. Roton-roton scattering was also examined by colliding roton beams.

The magnetic properties of liquid ${ }^{3} \mathrm{He}$ continue to intrigue several groups because ${ }^{3} \mathrm{He}$ is a model system with general interest. $\mathrm{M}$. Bravin et al. (CRBT, Grenoble) measured simultaneously the magnetization and heat release on the rapid melting of $80 \%$ polarized solid ${ }^{3} \mathrm{He}$. From this they found that the susceptibility decreases with increasing magnetism at 26 bar and $80 \mathrm{mK}$, so the expected metamagnetic transition predicted by Wollhard can be ruled out. One now waits to see if refinements to the theory may resolve the discrepancy. Meanwhile, precision experiments of the equilibrium magnetism at $30 \mathrm{~T}$ to confirm the result are underway.

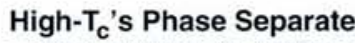

The manner in which antiferromagnetic high- $T_{c}$ superconductors on doping is of funlow-temperature section by M. Mehring (University of Stuttgart) reviewed the NMR evidence for phase separation in high- $T_{c}$ 's. Metallic behaviour and pronounced antiferromagnetic electron spin correlations still exist in superconducting Tl-cuprates so antiferromagnetic spin fluctuations clearly play an essential rôle in the superconducting pairing mechanism. Mehring also discussed NMR evidence for separation to antiferromagnetic (hole-poor) and metallic (electron-hole rich) components in in the various cuprate materials under different doping conditions. Neutron spectrosopic evidence for metallic and semiconducting clusters in the $\mathrm{CuO}_{2}$ planes of the $\mathrm{ErBaCuO}$ compound was discussed by $M$. Guillaume et al. (Zurich), highlighting the importance of spectroscopic techniques for local probing (see figure). The authors discussed how superconductivity can result from the formation of a two-dimensional bond percolation network, described theoretically by G. Siebold et al. (University of Stuttgart) in terms of spin-polarized clusters (polarons) formed on doping antiferromagnetically ordered high- $T_{c}$ cuprates with holes.

Another phase-separation phenomenon in high-T's, identified by magnetic measurements, involves microscopic segregation of doped holes into walls of hole-rich material lifetimes) at low temperatures. Above $50 \mathrm{mK}$, cuprate parent compounds evolve to become damental importance. An invited talk in the 
separating microscopic undoped domains in the antiferromagnetic phase found in a slightly non-stoichiometric LaSrCuO system. Larger non-stoichiometries bring about macroscopic phase separation to superconducting and non-superconducting phases.

\section{Renewed Interest in Quantum \\ Paraelectrics}

A semiconductors symposium on the existence of a coherent paraelectric state in $\mathrm{SrTiO}_{3}$ reflected the renewed interest in ferroelectric perovskites owing to the discovery of high-T $T_{c}$ superconductors. Specially interesting is the recently proposed low-temperature superfluid-like phase transition in quantum paraelectrics, where the physical characteristics of the phases remain unknown. Talks by groups representing the originators of the proposal (K.A. Müller, IBM Zurich, and E. Tosatti, SISSA, Trieste) discussed measurements of anomalous elastic properties, phonon anomalies from neutron and Brillouin scattering, and Monte Carlo studies.

\section{Reconstruction and Macroscopic Tunnelling}

The important discovery that the dynamics of flux vortices in high- $T_{C}$ superconductors in the force field of structural defects affects the zero-resistivity state has prompted much recent work as well as inspiring talks in the session on flux lines. D. Feinberg (CNRS, Grenoble) focussed on vortex structures, notably on the effect of extended defects as these appear particularly promising as pinning sites in $\mathrm{YBaCuO}$ compounds. It appears that these defects may lead to reconstruction of the vortex lattice with dramatic consequences on pinning properties and on the technologically important critical current.

R. Griessen (Free University, Amsterdam) described flux creep in several superconductors caused by what is believed to be macroscopic quantum tunnelling of vortices since there is a plateau in the magnetic relaxation rate below $1 \mathrm{~K}$. A giant quantum creep rate has been observed recently in a high- $\mathrm{T}_{\mathrm{c}}$ multilayer system, where identification of the tunnelling object was made possible by a

Giant quantum creep in high- $T_{C}$ multilayers. $A$ segment of a quantized flux vortex (as large as $300 \mathrm{~nm}$ in diameter parallel to the applied magnetic field) tunnels between adjacent configurations. (Courtesy of R. Griessen)

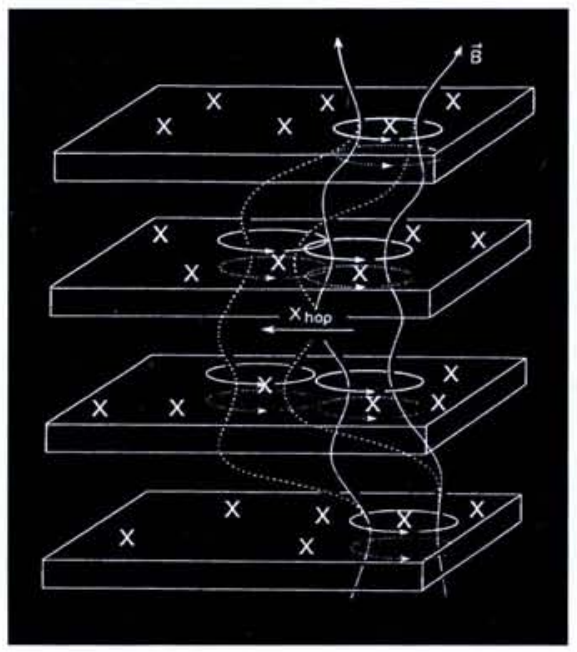

systematic study of the decay current in a high- $T_{c}$ multilayer system as a function of the thickness of one of the layers. The tunnelling object is surprisingly large - $300 \mathrm{~nm}$ in diameter and $4 \mathrm{~nm}$ in length - the latter in agreement with theories based on weak collective pinning. But mysteries remain. For instance, the relaxation rate, contrary to predictions, increases as the strength of an applied magnetic field increases. Second, measurements of the residual resistivity in high- $T_{c}$ superconductors show that the magnetic relaxation rate does not extrapolate to zero. This means either that Anderson's idea of a linear temperature dependence of the resistivity is wrong or dissipation is not related to the normal state resistivity, as assumed in existing quantum creep theories.

Another example of tunnelling between quantum states at the macroscopic level has recently been observed in magnetic systems by a group from Grenoble. The group discussed in an invited talk (B. Barbara et al.) how below typically $5 \mathrm{~K}$ the magnetization relaxation in amorphous magnetic multilayers remains constant and is thus not thermally activated. The reversal of magnetization in the non-interacting ferromagnetic layers can be interpreted in terms of quantum tunnelling of domain walls.

\section{Magnetic Multilayers Prominent}

Four sessions were devoted to magnetic multilayers and the main topic was undoubtedly the giant magnetoresistive effect observed at relatively low applied magnetic fields. Having considerable industrial significance, it originates from the totally unexpected appearance of oscillatory antiferromagnetic exchange coupling [see EN 24 (1993) 43]. Invited talks reviewed progress in the scientific understanding, with $P$. Brun (Université de Paris-Sud) describing a unified theoretical, easily visualised, description for calculating the long-range oscillatory coupling between ferromagnetic films accross a non-magnetic spacer. M.T Johnson (Eindhoven) summarised the latest experimental work for sandwiches grown by molecular beam epitaxy.

Of technological importance was a report by $R$. von Helmolt et al. (Siemens, University of Augsburg) that the giant magnetoresistance found recently in granular thin films also arises in more easily processed, rapidly solidified CuCo alloys containing nanocrystalline $\mathrm{Co}$ in a Cu matrix.

\section{Spectroscopy Using Polarized X-Rays Takes Off}

Many technologically important magnetic materials depend upon adjusting magnetic properties. This can only be done precisely if there exists direct information about the appropriate atomic shells which are the origin of these properties. By measuring differences in absorption (see figure) between well-defined left- and right-hand circularly polarized synchrotron $\mathrm{x}$-ray photon radiation (or between up and down, linearly polarized beams) one can estimate spin and orbital moments described in the framework of a one-electron model. The dichroism signal obtained by fluorescence detection of the absorption is proportional to the difference in the spin-up or spin-down occupation of a valence shell into which an electron is excited in the absorbing atom (i.e., proportional to the magnetic moment).

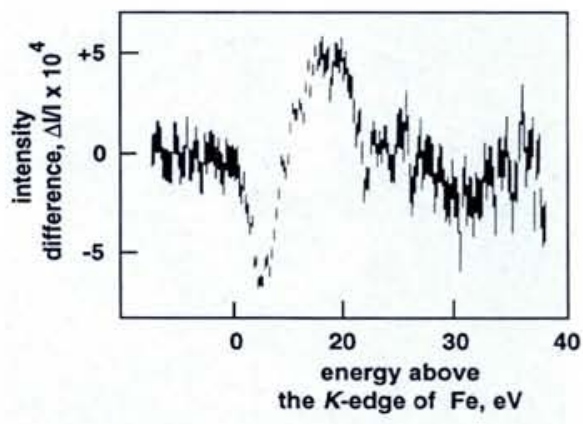

Spin-dependent photoabsorption of circularly polarized $x$-rays. The observed energy dependence, at energies above the K-absorption edge, of the difference between the transmission of $x$-rays circularly polarized in and opposite to the direction of the spin of magnetized $d$-electrons in a thin iron foil. [Schutz G., et al., Phys. Rev. Lett. 58 (1987) 737]

Refinements are on their way for this powerful new probe technique. For example, obtaining information from high-order adsorption edges requires high-energy $\mathrm{x}$-rays and a more complicated model (G. Krill, Université de Paris-Sud) or improved theoretical and experimental approaches if the usual soft $\mathrm{x}$-rays are used $(\mathrm{H}$. Ebert et al., Munich, Duisberg and IBM Almaden). G. Schütz (TU Munich) described the importance of magnetic $x$-ray dichroism for investigating the magnetic anistropy, originating from spinorbit interactions, of technologically important materials such as thin-film storage media.

$A$ related technique uses the detection of spin-polarized core-level photoelectrons, a new result being the first experimental evidence for the existence of a magnetic moment on the oxygen site in a magnetic oxide (M Knülle et al., TU Munich and BESSY, Hamburg). Backscattering of the outgoing spin-polarized photoelectrons by their spinpolarized neighbours provides a technique to probe the magnetic environments of nonmagnetic components (Ch. Roth et al., TU Munich).

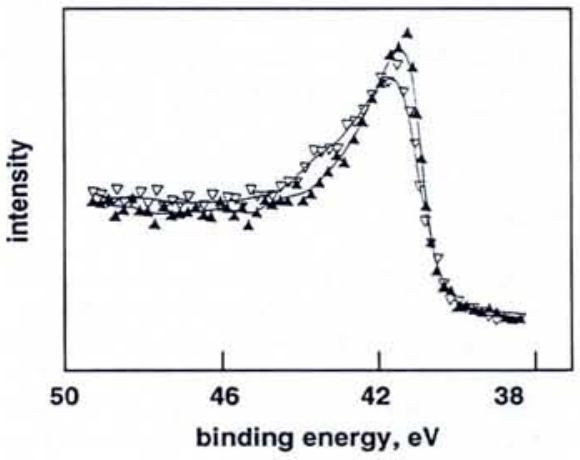

Spin-resolved core level photoemission of a thin $\mathrm{Cr}$ layer on $\mathrm{Fe}$. Synchrotron light emits photons that have moments parallel or antiparallel to the sample magnetization. The small energy separation between the two spin-resolved peaks of the Cr $3 p$ spectra arises owing to the interaction between the spin of the ionized shell and the spin of the valence electrons. There must therefore be ferromagnetic order in the $\mathrm{Cr}$ layer. [Hillebrecht F.U., et al., Europhys, Lett. 19 (1992) 711] 


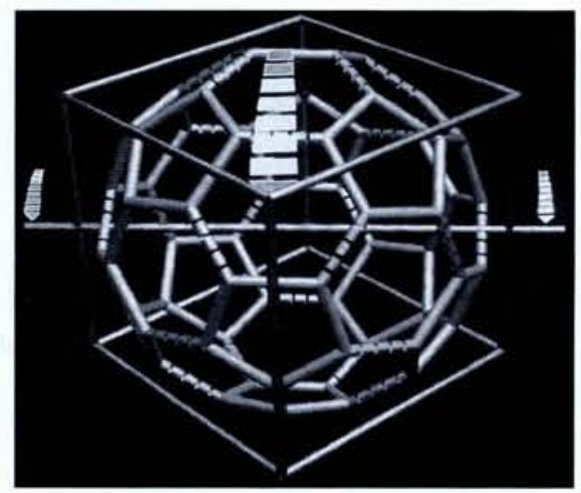

A representation of the quasi-isotropic reorientational motion of $C-C$ bonds in the ordered $C_{60}$ phase below $260 \mathrm{~K}$. The motion takes place about one of three symmetry-equivalent $<110>$ type directions (the arrows indicate the sense). [Courtesy of W.I.F. David]

\section{Fullerene Structures Take Shape}

Four sessions covered the fast-moving field of fullerenes and fullerites, while invited talks dealt with NMR studies of intercalated $\mathrm{C}_{60}$ (P. Bernier et al., University of Montpellier), crystal structures and transformations (W.I.F. David, Rutherford Appleton Laboratory), ab initio molecular dynamics calculations (W. Andreoni, IBM Zurich), structure and dynamics (K. Prassides, Sussex University), and microscopical theories of orientational ordering in solid $\mathrm{C}_{60}$ (K.H. Michel, University of Antwerp). NMR data confirm the existence of three non-equivalent carbon sites in doped $\mathrm{C}_{60}$ and thermodynamic data reveal possible precursor effects before the order-disorder transition at $260 \mathrm{~K}$ in undoped $\mathrm{C}_{60}$. More importantly, David was "99 percent sure" that the $\mathrm{C}_{60}$ molecule has an orientational glass transition at around $86 \mathrm{~K}$ that involves a single process comprising $42^{\circ}$ hops between energetically similar positions about one of three equivalent directions. $\mathrm{C}_{60}$ rotational order is represented as a spherical shell of scattering and somewhat surprisingly, maxima are along the three $<100>$ symmetry directions: the origin of extra scattering in $<111>$ directions is unclear.

The $a b$ initio molecular dynamics calculations are providing unique and detailed information on structural relaxation induced by confinement or doping effects. Meanwhile, millimetre-sized crystals of high purity $\mathrm{C}_{60}$ and $\mathrm{C}_{70}$ fullerenes have recently become available, thus allowing detailed studies of crystal structures, phase transitions, and crystal properties. Prassides reviewed phase transformations accompanying rotational ordering in $\mathrm{C}_{60}$ and its derivatives in the solid state where, for example, different orientationally ordered structures can be formed within the same positional polytype of $\mathrm{C}_{70}$ with the same stacking. He also examined the potential of using muons as spin labels and work along these lines reported by $\mathrm{C}$. Bernhard et al. (University of Constance) confirmed a slowing down of the rotational dynamics of $\mathrm{C}_{70}$ at low temperatures. A start has been made to describe orientational ordering in solid $\mathrm{C}_{60}$, notably by Michel who discussed his microscopic theory for the dynamics.

Reports of the many investigations of the fascinating properties of fullerites, somewhat scattered throughout several divisions, are simply too numerous to consider in detail. However, the BCS model of superconductivity still seems to be holding up as the critical temperatures of doped fullerenes with their carbon backbones approach $40 \mathrm{~K}$.

\section{Diamond and Ultra-Thin Films}

E. Wolfgangs's (Siemens) invited talk highlighted the importance of another novel form of carbon, namely high-conductivity diamond films, for coating semiconductor substrates to cope with the increasingly important problem of thermal dissipation in electronic devices. First demonstration models of devices suggest that the difference between the expansion coefficients of diamond and typical substrates can be accommodated.

The mechanical properties (e.g., elastic constants, sound velocities) of ultrathin films are technological important but measurement techniques are lacking, especially when the layers are too thin for Brillouin scattering. J.P. Toennies (Max-Plank, Göttingen) described inelastic helium atom scattering of clean and adsobate-covered metal surfaces, the latter revealing nearly dispersionless phonon modes corresponding to confined resonances which can be used to calculate elastic properties and even layer-by-layer relaxation.

\section{Persistent Currents Still Underestimated in Mesoscopic Metals}

A highlight of three invited talks and three sessions in the metals division on mesoscopic and microscopic systems was an invited talk by Y. Imry (Wiezmann Institute) on quantum interference phenomena found at low temperatures in normally conducting samples of mesoscopic size. The author reviewed orbital magnetism and persistent currents representative of low-dimensionality effects. The classical theory of spectral correlations for phase-coherent elastic scattering (with phase breaking processes such as inelastic scattering ignored or averaged) gives lower than predicted persistent currents in isolated rings in the presence of a magnetic field (U. Eckern, KFA Jülich). Attempts to explain this result in the free-electron approximation have failed. The current trend is to include electron-electron (or Coloumb interactions), in particular the rôle of local charge neutrality.

One direction for future applications stemming from fundamental research was hinted at by A.G. Aronov in his presentation accompanying the award of this year's HewlettPackard Europhysics Prize [see EN 24 (1993) 18]. It involves the recently reported work on electron waveguides comprising mesoscopic normal-metal rings with superconducting "mirror" boundaries.

\section{Semiconductor Mesoscopics for Atomic Models and New Devices}

The semiconductors and insulators division, notwithstanding considerable overlap with other divisions, remained by far the largest. Several major themes were highlighted, including transport in mesoscopic semiconductor structures in a symposium of invited talks. The trend is to observe electronic structures of increasingly smaller dimensionality to investigate the effect of dimensionality on properties. The amazing finesse that has been achieved was illustrated by science- fiction experiments (R.C. Ashoori, MIT) using capacitance spectroscopy to probe groundstate energy levels of an "artificial atom" comprising a quantum well in GaAs into which 1-50 electrons had been injected, and by L.W. Molenkamp (Philips) using a different technique (thermopower of a quantum point contact). In the case of a quantum dot, theory predicts that as the size decreases the energy level spacing becomes discrete. U. Shivan (Technion, Haifa) described experiments looking for this refinement effect, the goal being to model hydrogen and the like by seeing when cooperative phenomenon appear.

Once again, new directions involve combinations, this time between semiconductors and superconductors, to make novel mesoscopic devices. This work, lying at the interface between two huge fields, is in its infancy. B.J. van Wess (University of Groningen) discussed, for example, the behaviour of a short piece of a two-dimensional electron gas between classical superconductors where the electrons pass through the entire system without scattering.

\section{II-VI Quantum Wires May Hold the Future}

A major theme in semiconductor technology is the coming of age of II-VI optoelectronic devices. II-VI's have the fundamental advantage of being more polar than III-V's and the breakthrough stems from the fact that good quality doped materials can be gown using two-dimensional growth techniques. II-VI may hold the future for solidstate lasers: theory predicts there should be gains in lasing performance by moving from two- to one-dimensional confinement of electronic states, providing the amount of active material is not reduced too much. However, etching of III-V quantum well structures leads to reduced performance owing to passivation of the edges; this is not a problem for II-VI's.

The symposium of invited talks on the advances in the growth of II-VI quantum well structures included presentations by J. Cibert et al. (CNRS, Grenoble) on strained II-VI heterostructures, by W. Faschinger (University of Linz) on atomic layer epitaxial growth, and by B. Lunn (Hull University) on single layer and multiple quantum well structures.

\section{Precision Spectroscopies:}

\section{Some Promising, Some Disappointing}

Ultrafast time-resolved spectroscopy represents a tour-de-force but the output has been a little disappointing, with few new results in recent years. The appearance of good quality II-VI materials suggests this may change. Important theoretical contributions over several years describing non-equilibrium carrier dynamics ("hot carriers"), important for understanding the electronic properties of semiconductors, were reviewed by L. Reggiani (University of Modena) in a symposium on ultrafast processes.

Three-photon spectroscopy has developed these last few years to the stage that nonlinear processes in technically important, indirect-gap optical materials can be observed with enormous precision (e.g., $\mathrm{CuCl}$ : D. Fröhlich et al., University of Dortmund; $\mathrm{Cdl}_{2}$ : M. Lepore et al., University of Bari). The problem has been the lack of adequately intense lasers in a suitable warelength range. Detailed comparisons of experimental results with theory are now possible (see figure). 


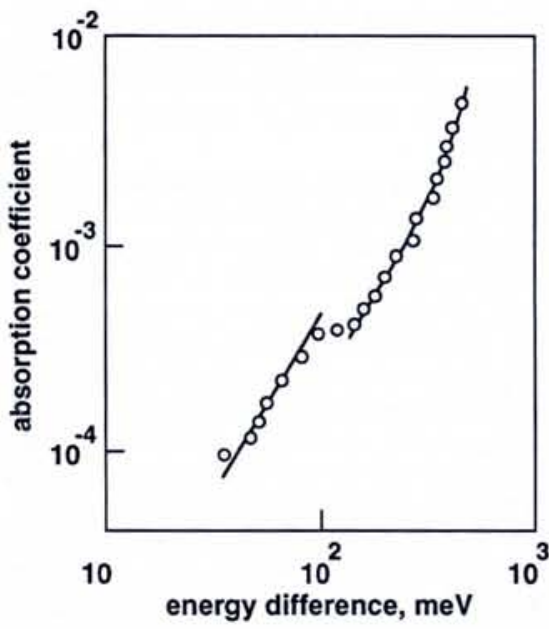

Observed three-photon absorption coefficients (points) of $\mathrm{Cdl}_{2}$ as a function of the energy difference relative to the indirect-gap energy. Theory cannot explain why the complex dependence is described by the two formulae (line and curve) used for direct-gap materials. [Lepore M., et al., Phys. Rev. B 47 (1993) 7580]

\section{Complex Systems:}

\section{Building Bridges and Localization}

Many well-known phenomena and substances which for many years physicists tended to put aside with the remark "not fundamental enough", maybe because the problems were too complex, have recently become tractable. The dynamics and statistical physics division highlighted studies of complex substances, complex systems, and soft matter. Other contributions addressed biological growth patterns, fracture, growth modes, growth dynamics, aggregation, and transport as well as composite media, porous materials, sandpiles, suspensions, and ionic crystals.

A major highlight was the invited talk by A.R. Bishop (Los Alamos National Laboratory) who presented a sweeping overview of how to build bridges between the microscopic and the macrosopic. The trick is to

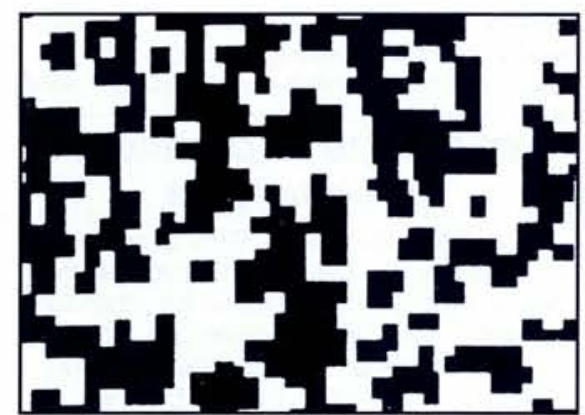

Relaxation at low temperatures of an initially random flux configuration, as simulated by a supercomputer for a $128 \times 128$ array of Josephson junctions in the presence of a magnetic field which frustrates the flux order. The checkerboard pattern of domain walls has frozen in after an initial relaxation. [GronbechJensen N., et al., Phys. Rev. B 45 (1992) 10139] use as building blocks for the mesoscopic and self-organized structure the space-time structures identified by the mathematics of soliton-bearing systems. Several two-dimensional condensed matter systems are now accessible by large-scale computation. As an example, two types of flux defects (domain walls and vortex/antivortex pairs) arise in rectangular arrays of Josephson junctions in a perpendicular magnetic field. Bishop illustrated how to develop phenomenologies for complex mesoscale order and dynamics by showing that the relaxation of domain walls can be used to diagnose mesoscopic order. The domains are pinned by the underlying frustration at low temperatures (see figure), but show a glass transition and crystallization at higher temperatures. One is in fact now in a position to test scaling in reciprocal space for the first time. Another goal will be to predict arrangements of the many two-dimensional defects (e.g., twins) found in complex crystalline phases.

There is currently considerable interest in the propagation and multiple scattering of light in disordered media with parameters that approach the threshold of where light becomes spatially localized in the presence of the disorder (Anderson localization). The topic was addressed in a series of three invited talks on the propagation of waves and electrons in disordered media (A. Lagendijk, FOM Amsterdam), on the effect of magnetic fields (G. Maret, CNRS, Grenoble), and on a technique called diffusing wave spectroscopy which uses dynamic light scattering to study materials which are in fact optically opaque (D.A. Weitz, Exxon).

\section{A Universal Glass Parameter and} $\mathrm{H}^{-}$Conductors

Concerning neutron scattering, presentations by V.K. Malinovsky et al. (Novosibirsk) and A.P. Sokolov et al. (Free University, Berlin) argued that glasses have universal medium-range order on a scale of about $1 \mathrm{~nm}$ which accounts, for example, for the low-temperature density of vibrational states for glasses in the same way as Debye's law for crystals. The model is claimed to have important implications for descriptions of phenomena arising in glasses such as the effect of Anderson localization of excitations on energy transfer kinetics.

Finally, a neutron scattering group ( $F$. Altorfer et al.) using the Saclay facility showed that $\mathrm{H}^{-}$diffuses in $\mathrm{Ba}_{2} \mathrm{NH}$ (recently reported to be the first documented $\mathrm{H}^{-}$ion conductor) by two-dimensional hopping on regular lattice sites.

The highlights of the Regensburg meeting clearly spanned major industrial applications of new discoveries to the basic understanding of fundamental phenomena, while at the same time throwing up a number of intriguing new ideas, new materials, and new techniques. The proceedings, containing probably around $80 \%$ of the 179 invited and plenary talks, will be published by November 1993 in two volumes by Physica Scripta. Next year's CMD General Conference in Lisbon on 28-31 March will not be on quite the same scale. But one can be sure that it will highlight its fair share of new physics across what has become a huge field.

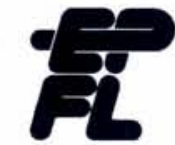

\section{Swiss Federal Institute of Technology - Lausanne}

Applications are invited for a position of

\section{Assistant-Professor in Plasma Physics}

at the Centre for Plasma Physics Research.

The successful applicant will participate in the research programme of the Centre in the field of Plasma Physics applied to Magnetic Fusion, and is expected to contribute to teaching at the undergraduate and graduate level through the Physics Department of the EPFL.

The successful candidate will enter into service from Autumn 1993.

Interested candidates should request the full dossier concerning this position at the following address:

Présidence de l'Ecole Polytechnique Fédérale de Lausanne,

CE-Ecublens,

$\mathrm{CH}$ - 1015 Lausanne, Switzerland. 